\title{
Weather sensitivity in migraineurs
}

\author{
Jan Hoffmann · Hendra Lo • Lars Neeb • \\ Peter Martus $\cdot$ Uwe Reuter
}

Received: 24 June 2010/Revised: 18 September 2010/Accepted: 7 October 2010/Published online: 24 October 2010

(C) The Author(s) 2010. This article is published with open access at Springerlink.com

\begin{abstract}
The scientific evidence for weather being a trigger factor for migraine attacks is inconclusive. We investigated the association between weather components and the onset and severity of attacks. Headache diaries of 20 migraineurs were analyzed retrospectively and correlated in 4-h intervals to atmospheric air pressure, temperature, and relative air humidity in Berlin (Germany) for a period of 12 consecutive months. Absolute values and relative changes within the preceding $24 \mathrm{~h}$ were analyzed. Migraine attacks started most frequently at 4 a.m. and reached the highest intensity between 4 and 8 a.m. A highly significant association between meteorological variables and the occurrence of migraine attacks was found in six patients. The onset of an attack as well as high headache intensity was associated with lower temperature and higher humidity. Our data indicate that a subgroup of migraineurs is highly sensitive to changes of certain weather components.
\end{abstract}

P. Martus and U. Reuter contributed equally to the manuscript.

J. Hoffmann · H. Lo $\cdot$ L. Neeb · U. Reuter $(\bowtie)$

Department of Neurology, Charité-Universitätsmedizin Berlin, Charitéplatz 1, 10117 Berlin, Germany

e-mail: uwe.reuter@charite.de

\section{J. Hoffmann}

Department of Neurology, University of California,

San Francisco, 505 Parnassus Avenue,

San Francisco, CA 94143, USA

\section{P. Martus}

Institute of Biometry and Clinical Epidemiology, Charité-Universitätsmedizin Berlin, Charitéplatz 1, 10117 Berlin, Germany
Keywords Migraine - Weather - Temperature . Humidity $\cdot$ Atmospheric pressure

\section{Introduction}

The link between migraine and weather remains obscure. While some migraineurs convincingly report weather as a reliable exogenous trigger factor for their attacks, others strictly rule out any weather influence. Clinical studies support these contradicting observations [1].

Epidemiological analyses were also not conclusive so far. Cooke et al. [2] found a higher probability for the start of a migraine attack on preChinook and Chinook wind days in different patient subgroups in western Canada. In addition, $50 \%$ of subjects were found to be weather sensitive in a North American prospective study [3]. However, emergency room treatments for migraine were not correlated with weather conditions $24 \mathrm{~h}$ prior to the visit which raises doubts about an influence of the weather on severe migraine attacks, in line with results from another British study [4, 5]. This analysis failed to identify a relationship between atmospheric changes and the incidence of migraine. A separate group of 100 patients attending the Princess Margaret Migraine Clinic in London for routine consultation was analyzed with the same negative result [5]. Several reasons may account for the differences between studies.

The study of weather as a causal factor of migraine is further hampered by a number of complexities. Weather consists of several components such as air pressure, humidity, temperature, and their changes from day to day and within 1 day. Moreover, wind and spherics also vary within hours. Therefore, each of these factors must be analyzed separately in relation to migraine and daytime has to be taken into account as a possible confounding factor. 
The aim of our study was to analyze headache data over a period of 12 consecutive months and correlate these to specific weather components and their relative changes in order to determine whether any of these factors is linked to the occurrence or severity of a migraine attack.

\section{Methods}

Patients

We retrospectively evaluated the clinical data of 20 randomly selected patients ( 5 male, 15 female) of the Headache Outpatient Department at Charité Universitätsmedizin Berlin, Germany. For inclusion, patients had to be 18 to 65-years-old, residents of Berlin (max. $50 \mathrm{~km}$ distance from the headache center) and diagnosed with episodic migraine with or without aura (migraine with aura $n=4$, migraine without aura $n=16$ ) based on the criteria of the International Headache Society [6]. We asked consecutive patients who presented for routine consultation and also fulfilled the inclusion criteria for consent. If positive, the headache diaries of the preceding 12 months of each patient were included in the study until a total of 20 calendar sets for analysis was reached.

Data were acquired from the patient's headache diary, which is routinely used by all patients of the headache clinic. Herein, patients report headache frequency, location, duration, character, and intensity in 4-h intervals. Headache intensity is rated on a scale between 0 (no headache) and 5 (max. intensity). Headache diaries had to be completed over at least 12 consecutive months between 1 January 2006 and 31 December 2007. The 12-month time frame was chosen in order to exclude a possible bias that may be caused by seasonal differences of migraine attack occurrence. Patients with any headaches other than migraine were excluded from the study. Data of patients on prophylactic migraine treatment were included if the patient was on a stable dose for at least 3 months prior to and during the entire observation period.

\section{Weather data}

Weather data were obtained from the German Meteorological Service (Deutscher Wetterdienst, Offenbach, Germany) for the meteorological station Berlin-Alexanderplatz (distance to hospital approx. $700 \mathrm{~m}$ ). Weather data were recorded every single hour, $24 \mathrm{~h}$ a day from 1 January 2006 to 31 December 2007. Statistical analysis used the data in 4-h time frames in analogy to the patient diaries. Data were obtained for atmospheric pressure (in $\mathrm{HPa}$ ), temperature (in ${ }^{\circ} \mathrm{C}$ ) and relative air humidity (in \%).
Statistics

We differentiate between migraine attacks and migraine periods. Migraine attacks were documented by patient diaries. An attack is a single note in this diary. Single attacks were combined to migraine periods. Two attacks were defined as belonging to two separate migraine periods if the headache free interval between both attacks was at least $48 \mathrm{~h}$. The beginning of migraine periods and the intensity of migraine attacks were correlated with meteorological data from the preceding $24 \mathrm{~h}$. Absolute values of these data as well as changes were taken into account. For each patient a separate analysis of 1-year data was performed. This approach required calculations with a total of 788.400 data points. In order to avoid multiple testing for the calendar analyses of months, days, and time of day, pairwise comparisons were done using Tukey's $B$ test. This type of test is suitable when all pairwise tests are performed for any specific factor. For the construction of classifiers (individually for the patients), logistic regression analysis (outcome: migraine period yes/no; covariates: meteorological data, calendar data, diurnal profile data) was applied with forward variable selection. Forward variable selection is a technique which avoids the overfitting of models, i.e., the inclusion of too many covariates. Quality of classification was examined using ROC analysis. These results were cross validated using the leaving-one-out method. The underlying idea is to construct 20 different classifiers, ignoring one patient for each. Subsequently, each of these classifiers is applied to exactly that subject, which was not used for the construction of the classifier. This leads to an unbiased estimation of the true classification rate. The level of significance was 0.05 (two-sided). As multiple significance tests were applied, the number of significant results expected by chance and the observed number of significant results were compared. Overall, 420 statistical tests were applied (20 subjects, 3 variables, 7 measurement points) in the analysis of migraine intensities. To adjust for multiple testing the method of false discovery rate (FDR) was applied for an alpha error of 0.05 [7]. This method is standard in bioinformatics where large numbers of tests are very common. Using this method the $p$ values are ordered for decreasing values. The highest $p$ value is compared to 0.05 , the second highest to $0.05 / 2$, and so on, the smallest $p$ value is compared to $0.05 / 420$. If in this sequence for one corrected $p$ value significance is achieved, all $p$ values smaller than this index value are considered significant. It is then ensured, that the frequency of falsely rejected null hypotheses is at most $5 \%$. For statistical calculations commercially available software (SPSS for Windows, 15.0) was used. 


\section{Results}

Calendar data

Data analysis revealed highly significant differences between the six time points defined by the 4-h intervals for the onset of migraine ( $p<0.001$ for attacks and periods). Migraine periods started more frequently at 4 a.m. as compared to all other time points (Fig. 1). They were most severe at onset $(4 \mathrm{a} . \mathrm{m}$.) and at 8 a.m. and less severe at midnight (Fig. 2).

There was a slight dependency of migraine attacks on months, with a peak number in January and less frequent attacks in August; however, this was not statistically significant (Fig. 3). We did not find a relation between specific days of the week (e.g., weekend) and migraine attacks.

Weather data, patient-wise analysis adjusted for time of the day

Daytime adjustment was necessary as the probability of migraine varies significantly during the course of a day. After adjusting for daytime, 106 significant results were found in 780 significance tests (number expected by chance: 39 significant results). In six patients (patient ID 3, $4,9,11,15,20$ ), at least seven significant associations were found between meteorological variables and headache intensities compared to two significances expected by chance (Table 1). In another six patients none, one, or two significances were observed (patient ID 2, 6, 8, 12, 13, 18, Table 1). For the remaining patients, three to six significant results were found. Concerning the three types of meteorological measurements, $n=34$ significances were found for air pressure, $n=28$ for temperature, and $n=44$ for relative humidity (for each type of meteorological variable,

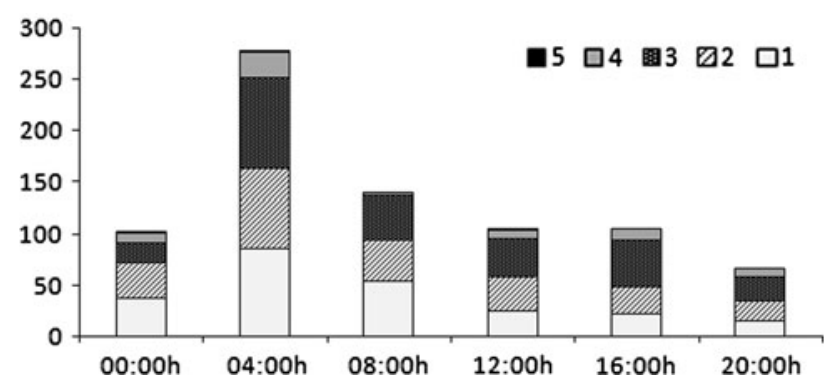

Fig. 1 The time of onset of new migraine periods (first headache diary entry of a migraine attack after a headache free period of at least $48 \mathrm{~h}$ ). The colour code shows the intensities of the first headache diary entry (darker colours indicate strong headaches and light colours indicate milder headache). The start of more than 250 new migraine periods was recorded at 4 a.m., which is strikingly different from all other time points. As expected, the lowest number of new migraine periods $(n=65)$ began in the evening ( 8 p.m.)

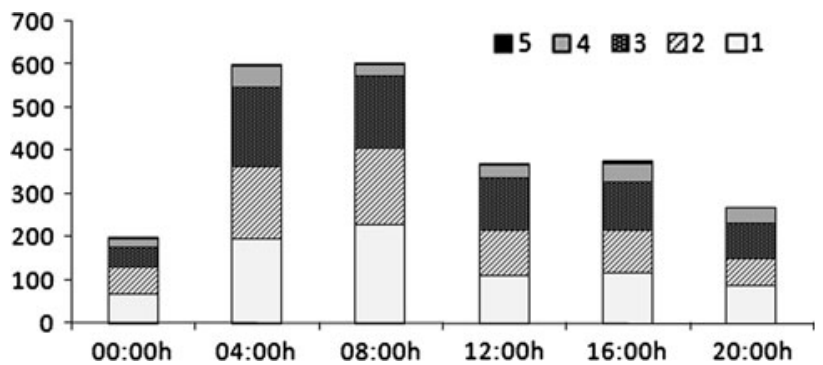

Fig. 2 The intensities of all migraine attacks according to daytime (all entries are analyzed). Migraine attacks were most intense in the morning between 4 and 8 a.m. Few entries are recorded at midnight, which might be due to nocturnal sleep. However, if headaches were severe at this time point, subjects would wake up due to the pain as might be the case for headache onset at 4 a.m. (as illustrated in Fig. 1)

13 significances would have been expected). Applying the FDR method, 23 significant tests out of 420 FDR tests remained. Among these tests, 21 were related to four of the 20 subjects (subject number $3,4,9$, and 20). The significant tests were equally distributed for the time points, 12 out of 23 significances were obtained for air pressure values. The remaining significances were equally distributed between humidity (six significant results) and temperature (five significant results).

Weather data, patient-wise classification, adjusted for time of the day

For five patients (ID 4, 5, 9, 15, 20), an individual classification for the occurrence of migraine periods according to weather data was feasible. For these patients, areas under the curve (cross validated) were between 0.72 and 0.82 . Note that this classification was based on weather variables combined with time of the day. Areas under the ROC curve for only time of the day were about $5 \%$ smaller than those including both, weather data and time of the day. There was no correlation between the number of migraine periods or attacks and the sensitivity to any weather component.

Weather data, analysis for the overall population

No significant association was found between air pressure and the beginning of new migraine periods. In contrast, temperature was significantly lower and humidity was significantly higher at the beginning of periods. Similarly, lower temperature and higher humidity were associated with a higher intensity of attacks. After adjustment for daytime, a weak association between temperature (18 and $24 \mathrm{~h}$ before the period) and onset of a new migraine period persisted. In addition, the intensity of attacks and lower air pressure were significantly associated as well as headache intensity and humidity. A marginal association was observed between the intensity of attacks and temperature. 
Fig. 3 The absolute number of all migraine attacks within 12 months. While migraine occurs most frequently in January, the number of attacks was lower in August. However, a clear relation of attacks to any month could not be detected

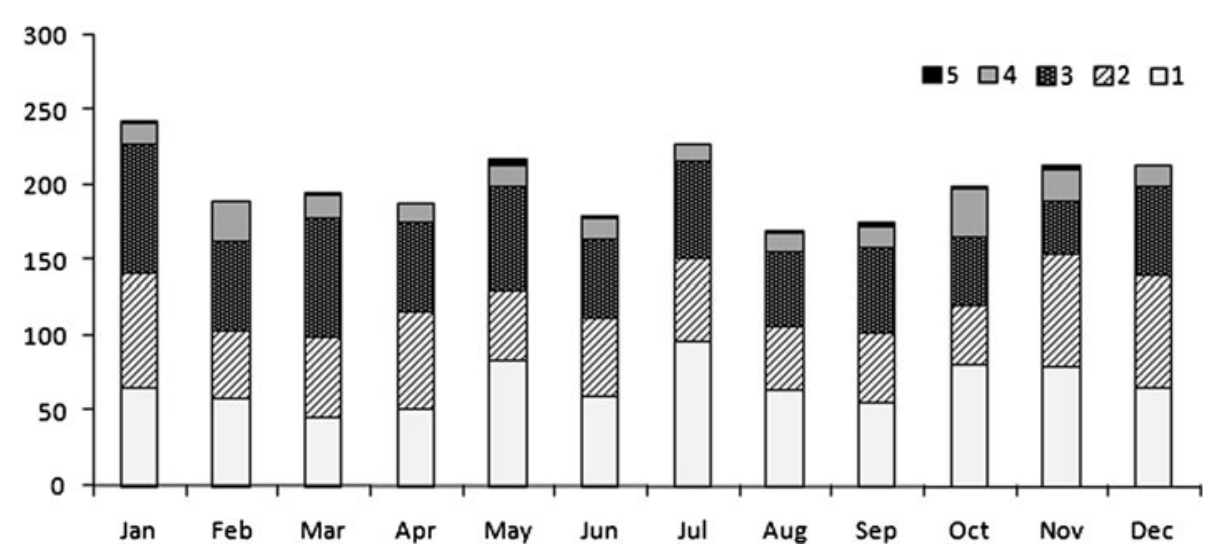

The area under the ROC curve was 0.57 in multivariate analysis (cross validated) and thus not satisfactorily.

\section{Discussion}

Our study shows that, in a subset of patients, lower temperature and higher relative humidity correlate with the onset of a migraine period independently of the time of the day. In some patients an individual classification for the occurrence of migraine periods according to weather data was feasible. Highly significant associations were found between relative air humidity as well as atmospheric air pressure and headache intensities in a subset of individuals. In only four patients no correlation to any of the investigated weather parameters was seen. We conclude that a subgroup of migraine subjects is sensitive to changes in temperature and relative humidity which results in the generation of a migraine attack.

Two older studies did not find any significant correlation between the occurrence of migraine attacks and atmospheric pressure, temperature, or relative humidity [5, 8]. Likewise, a more recent study which investigated the correlation between changes of specific weather parameters and the number of emergency room visits for treatment of an acute migraine attack, failed to prove a direct link [4].

A new analysis of 7,054 emergency room patients with the primary discharge diagnosis of "headache" showed that higher ambient temperature leads to an increased risk of headache requiring emergency department evaluation. The risk further increases in migraineurs. Interestingly, atmospheric air pressure only increased the headache risk of non-migraine cases [9]. Despite the high number of patients evaluated in this study, results have to be interpreted with caution as the diagnosis was made by emergency department physicians not specialized in neurology and based only on a single consultation. The onset of headache was also not documented in this study.
Contradicting findings exist. Cooke et al. $[2,10]$ could identify an association between the occurrence of migraine attacks and Chinook winds. Chinook winds occur in the southern part of the province of Alberta, Canada, and are associated with a drop in atmospheric pressure as the wind starts blowing high wind velocities and an abrupt increase in temperature. For a subgroup of patients (15 out of 75), a significant correlation was identified. Another prospective North American study observed a correlation between migraine and specific weather parameters in a subgroup of patients [3]. Cull et al. [11] found that lower atmospheric pressure leads to a lower attack frequency, a finding which could not be reproduced by Cooke et al. [2].

Many reasons may account for different results between previous studies. In particular, prior to the IHS classification 1988, study inclusion and observation criteria were soft and imprecise. Primary headache disorders were not analyzed separately which resulted in the combined analysis of disorders with different underlying pathophysiological mechanisms $[12,13]$. This led to non reproducibility of findings. Various weather components (e.g., atmospheric pressure, temperature, relative humidity) were often analyzed together in relation to the occurrence of migraine attacks [1]. In studies with separate analyses of specific weather components, various classifiers were used which also reduces comparability.

While prospective data acquisition is usually preferred in clinical studies, the retrospective design of our analysis has the advantage that headache recordings are not biased because patients were not aware of the study when they documented their migraines. Moreover, we analyzed three specific meteorological parameters and used a small time frame of 4-h intervals for the comparison with data obtained from the headache diaries.

Another advantage of our study is the methodological approach. We evaluated headache data of each patient in a longitudinal approach. This approach was necessary to determine whether the suspected sensitivity to specific 


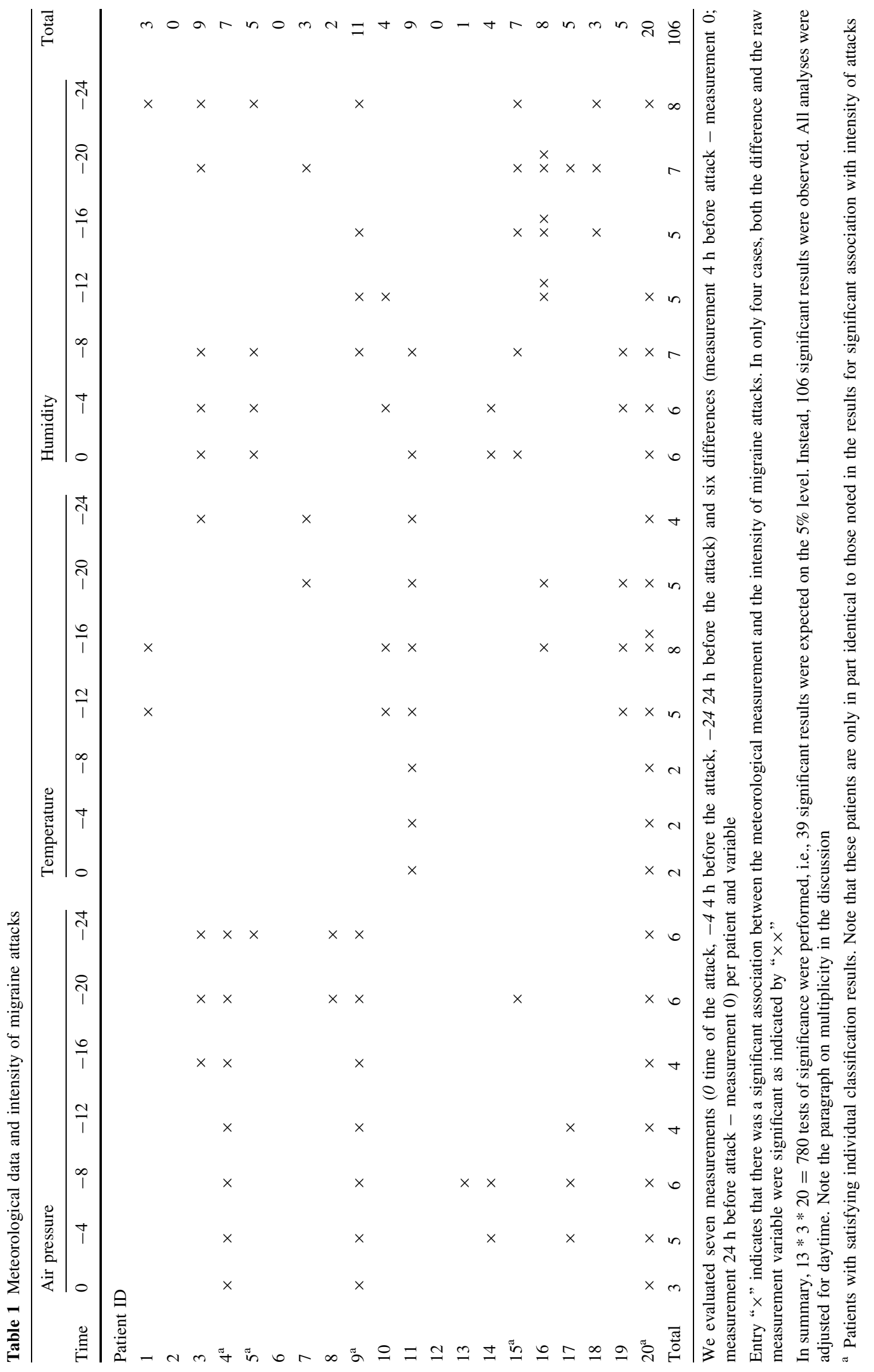


meteorological parameters affects all or only a subgroup of migraineurs. Hence, our statistical approach allows us to correlate the onset of migraine attacks to a certain weather condition and, beyond that, to consider the preceding $24 \mathrm{~h}$ in the analysis. To the best of our knowledge, a migraine weather study with a longitudinal approach which includes three weather parameters and observation time frames as short as $4 \mathrm{~h}$ has not been published.

The patient-wise analysis of our study might, at least in part, explain why we reach statistical significance for some parameters while others failed to do so, especially if significance was calculated for the entire population.

Potential shortcomings of this work are mainly of a statistical nature and result from the huge amount of data points. Because of the quantity of headache (and weather parameter) time points, even slight differences quickly reach statistical significance. These significances, as such, do not always permit conclusions and might even be far away from resembling clinical reality. To address this problem we performed multiple testing. Obviously this approach (multiple testing) is sensitive to the problems associated with multiple testing.

We performed 780 tests of significance (Table 1) and, therefore, expected 39 significant results by chance. However, 106 significant results were detected. In addition, some patients show a strikingly clear sensitivity to meteorological data. Even if we exclude patient 20 (with outstanding correlations) from the analysis, 86 significant results still remain in contrast to the 37 expected ones.

We also found some patients who show only few or no significant results. Thus, our analysis suggests that some patients are clearly not sensitive to meteorological conditions.

Our analysis could be hampered by the fact that the variables used in the tests were in part highly correlated, especially due to the high autocorrelation in the three time series of meteorological data. However, this does not change the number of expected significances but may lead to more (or less) significances by chance.

In an exploratory principal component analysis (results not given in detail), five components account for $93 \%$ of the variance of our meteorological variables (criterion Eigen value $>1$ ). Thus, we can assume that the meteorological data contain five independent pieces of information for each of the 20 patients. With 100 independent significance tests, five significances would have been expected, and the upper limit of a two-sided $95 \%$ confidence interval for the number of observed significances would have been $11.28 \%$. We found 106 in $780=13.6 \%$ significant results which may be an argument that overall results did not appear by chance. Because of the extensive statistical analysis it is obvious that no single significant result of our study confirms the hypothesis. On the other hand, lack of significance by no means proves absence of effect. From our point of view the overall results show proof of concept.

One shortcoming is the relatively small sample number. Nevertheless, even with 20 patients it seems to be evident that there are weather-sensitive and weather-insensitive migraineurs. This finding is unlikely to change even with a higher amount of patients. Because only a subset of patients is weather sensitive, we cannot rule out entirely that a potential barometric effect may appear in a significantly larger sample size.

However, the presented sample size does not permit reliable individual predictions about a single weatherinduced migraine attack.

We can only speculate about the effect of preventatives on this analysis. As preventatives reduce the probability of attacks triggered by different stimuli, their use could possibly confound the analysis, i.e., reducing the strength of any causal effect. To minimize potential confounding effects of preventatives, all patients had to remain on a stable dose throughout the course of the study.

In this study we are focussing on headaches as a key feature of a migraine attack. Other components of migraine, such as autonomous symptoms, aura or premonitory symptoms, were not studied. This may be a shortcoming. However, we concentrated on the migraine headache as the most frequent symptom in order to generate a result that applies to the vast majority of patients. The study of premonitory symptoms in relation to the weather is interesting, but is confounded by the frequency and character of symptoms. Many patients are not aware that a certain symptom (e.g., mood swings, strong appetite) which proceeds the headache is a part of their migraine or only realize post hoc once the headache occurs. Accordingly, patients' diaries are incomplete in this respect and it is almost impossible to run a retrospective analysis.

The pathophysiological link between weather changes and the occurrence of migraine attacks remains obscure and our analysis is not intended to provide insight into this matter. One may speculate that certain changes of specific weather parameters lead to an increase of neuronal excitability of trigeminal neurons and thereby facilitate the beginning of a migraine attack. The reason why this association can be found only in a subgroup of migraineurs remains unclear. Genetic predisposition may be the cause for higher susceptibility to neuron-exciting weather parameters.

In summary, our data demonstrate that in a significant subset of migraineurs the change of specific weather components is associated with the onset of a migraine attack. This finding suggests that affected migraineurs have an increased susceptibility to these weather conditions. 
Acknowledgments The authors would like to thank the German Meteorological Service (Deutscher Wetterdienst-DWD) for providing the weather data.

Conflict of interest The authors declare that they have no conflict of interest.

Open Access This article is distributed under the terms of the Creative Commons Attribution Noncommercial License which permits any noncommercial use, distribution, and reproduction in any medium, provided the original author(s) and source are credited.

\section{References}

1. Kelman L (2007) The triggers or precipitants of the acute migraine attack. Cephalalgia 27:394-402

2. Cooke LJ, Rose MS, Becker WJ (2000) Chinook winds and migraine headache. Neurology 54:302-307

3. Prince PB, Rapoport AM, Sheftell FD, Tepper SJ, Bigal ME (2004) The effect of weather on headache. Headache 44:596-602

4. Villeneuve PJ, Szyszkowicz M, Stieb D, Bourque DA (2006) Weather and emergency room visits for migraine headaches in Ottawa, Canada. Headache 46:64-72
5. Wilkinson M, Woodrow J (1979) Migraine and weather. Headache 19:375-378

6. Headache Classification Subcommittee of the International Headache Society (2004) The international classification of headache disorders (second edition). Cephalalgia 24(Suppl 1): $1-151$

7. Benjamini Y, Hochberg Y (1995) Controlling the false discovery rate: a practical and powerful approach to multiple testing. J R Statist Soc B 57(1):289-300

8. Gomersall JD, Stuart A (1973) Variations in migraine attacks with changes in weather conditions. Int J Biometeor 17:285-299

9. Mukamal KJ, Wellenius GA, Suh HH, Mittleman MA (2009) Weather and air pollution as triggers of severe headaches. Neurology 72:922-927

10. Piorecky J, Becker WJ, Rose MS (1997) Effect of Chinook winds on the probability of migraine headache occurrence. Headache 37:153-158

11. Cull RE (1981) Barometric pressure and other factors in migraine. Headache 21:102-104

12. Schulman J, Leviton A, Slack W, Porter D, Graham JR (1980) The relationship between headache occurence to barometric pressure. Int J Biometeor 24:263-269

13. Osterman PO, Lövstrand KG, Lundberg PO, Lundquist S, Muhr C (1981) Weekly headache periodicity and the effect of weather changes on headache. Int J Biometeor 25:39-45 